\title{
Inverse-Exponential Correlation Bounds and Extremely Rigid Matrices from a New Derandomized XOR Lemma
}

\author{
Lijie Chen \\ MIT, USA \\ lijieche@mit.edu
}

\begin{abstract}
In this work we prove that there is a function $f \in \mathrm{E}^{\mathrm{NP}}$ such that, for every sufficiently large $n$ and $d=\sqrt{n} / \log n, f_{n}$ ( $f$ restricted to $n$-bit inputs) cannot be $\left(1 / 2+2^{-d}\right)$-approximated by $\mathbb{F}_{2}$-polynomials of degree $d$. We also observe that a minor improvement (e.g., improving $d$ to $n^{1 / 2+\varepsilon}$ for any $\varepsilon>0$ ) over our result would imply $\mathrm{E}^{\mathrm{NP}}$ cannot be computed by depth- $3 \mathrm{AC}^{0}$-circuits of $2^{n^{1 / 2+\varepsilon}}$ size, which is a notoriously hard open question in complexity theory.

Using the same proof techniques, we are also able to construct extremely rigid matrices over $\mathbb{F}_{2}$ in $\mathrm{P}^{\mathrm{NP}}$. More specifically, we show that for every constant $\varepsilon \in(0,1)$, there is a $\mathrm{P}^{\mathrm{NP}}$ algorithm which on input $1^{n}$ outputs an $n \times n \mathbb{F}_{2}$-matrix $H_{n}$ satisfying $\mathcal{R}_{H_{n}}\left(2^{\log ^{1-\varepsilon} n}\right) \geq$ $\left(1 / 2-\exp \left(-\log ^{2 / 3 \cdot \varepsilon} n\right)\right) \cdot n^{2}$, for every sufficiently large $n$. This improves the recent $\mathrm{P}^{\mathrm{NP}}$ constructions of rigid matrices in [Alman and Chen, FOCS 2019] and [Bhangale et al., FOCS 2020], which only give $\Omega\left(n^{2}\right)$ rigidity.

The key ingredient in the proof of our new results is a new derandomized XOR lemma based on approximate linear sums, which roughly says that given an $n$-input function $f$ which cannot be 0.99-approximated by certain linear sum of $s$ many functions in $\mathcal{F}$ within $\ell_{1}$-distance, one can construct a new function $\mathrm{Amp}^{f}$ with $\widetilde{O}(n)$ input bits, which cannot be $\left(1 / 2+s^{\Omega(1)}\right)$-approximated by $\mathcal{F}$-functions. Taking $\mathcal{F}$ to be a function collection containing lowdegree $\mathbb{F}_{2}$-polynomials or low-rank $\mathbb{F}_{2}$-matrices, our results are then obtained by first using the algorithmic method to construct a function which is weakly hard against linear sums of $\mathcal{F}$ in the above sense, and then applying the derandomized XOR lemma to $f$.

We obtain our new derandomized XOR lemma by giving a generalization of the famous hardcore lemma by Impagliazzo. Our generalization in some sense constructs a non-Boolean hardcore of a weakly hard function $f$ with respect to $\mathcal{F}$-functions, from the weak inapproximability of $f$ by any linear sum of $\mathcal{F}$ with bounded $\ell_{p}$-norm. This generalization recovers the original hardcore lemma by considering the $\ell_{\infty}$-norm. Surprisingly, when we switch to the $\ell_{1}$-norm, we immediately rediscover Levin's proof of Yao's XOR Lemma. That is, these first two proofs of Yao's XOR Lemma can be unified with our new perspective. For proving the correlation bounds, our new derandomized XOR lemma indeed works with the $\ell_{4 / 3}$-norm.
\end{abstract}

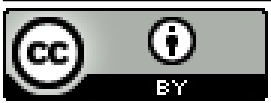

This work is licensed under a Creative Commons Attribution International 4.0 License. STOC '21, fune 21-25, 2021, Virtual, Italy

(C) 2021 Copyright held by the owner/author(s).

ACM ISBN 978-1-4503-8053-9/21/06.

https://doi.org/10.1145/3406325.3451132

\author{
Xin Lyu \\ Tsinghua University, China \\ lvx17@mails.tsinghua.edu.cn
}

\section{CCS CONCEPTS}

- Theory of computation $\rightarrow$ Circuit complexity; Complexity classes; Pseudorandomness and derandomization.

\section{KEYWORDS}

Correlation Bounds, rigidity, XOR Lemmas

ACM Reference Format:

Lijie Chen and Xin Lyu. 2021. Inverse-Exponential Correlation Bounds and Extremely Rigid Matrices from a New Derandomized XOR Lemma. In Proceedings of the 53rd Annual ACM SIGACT Symposium on Theory of Computing (STOC '21), June 21-25, 2021, Virtual, Italy. ACM, New York, NY, USA, 11 pages. https://doi.org/10.1145/3406325.3451132

\section{INTRODUCTION}

\subsection{Background}

We consider multivariate polynomials over $\mathbb{F}_{2}$. In particular, every $n$-variable polynomial $P:\{0,1\}^{n} \rightarrow\{0,1\}$ over $\mathbb{F}_{2}$ can be written as

$$
P\left(x_{1}, \ldots, x_{n}\right) \equiv \sum_{S \subseteq[n]} \alpha_{S} \cdot \prod_{i \in S} x_{i} . \quad(\bmod 2)
$$

The degree of $P$, denoted as $\operatorname{deg}(P)$, is defined as $\max \left\{|S|: \alpha_{S} \neq 0\right\}$.

Understanding the power and limitations of $\mathbb{F}_{2}$-polynomials as a model of computation is of fundamental interest in complexity theory. We will be particularly interested in exhibiting functions which are hard to approximate by low-degree $\mathbb{F}_{2}$-polynomials. ${ }^{1}$ Formally, for any two functions $f, g:\{0,1\}^{n} \rightarrow\{0,1\}$, we define their correlation as

$$
\operatorname{corr}(f, g)=\left|\operatorname{Pr}_{x \leftarrow U_{n}}[f(x)=g(x)]-\operatorname{Pr}_{x \leftarrow U_{n}}[f(x) \neq g(x)]\right| .
$$

Let $\mathcal{P}_{d}$ be the collection of $\mathbb{F}_{2}$-polynomials with degree $\leq d$. Slightly abusing notation, we also write $\operatorname{corr}(f, d):=\max _{P \in \mathcal{P}_{d}} \operatorname{corr}(f, P)$.

The correlation bound against $\mathbb{F}_{2}$-polynomials ${ }^{2}$, a major question in complexity theory, asks to find an explicit function (i.e., a function in NP) which cannot be approximated by low-degree $\mathbb{F}_{2}$-polynomials, that is, to prove upper bounds on $\operatorname{corr}(f, d)$ for an explicit function $f$ and various choices of $d$.

Since $\mathbb{F}_{2}$-polynomial is such a simple and elegant computational model, this question is clearly interesting in its own right. It is nonetheless also inherently connected to a variety of other fundamental questions in complexity theory. In fact, it was shown that improvements on correlation bound against $\mathbb{F}_{2}$-polynomials are necessary or sufficient for progress on other major open questions.

\footnotetext{
${ }^{1}$ We remark that it is not hard to exhibit a function which cannot be exactly computed by $\mathbb{F}_{2}$-polynomials of degree less than $n$. An example would be the AND function on $n$ input bits.

${ }^{2}$ In the Boolean setting, correlation bounds and average-case lower bounds are equivalent, and we will use these two terms interchangeably in this paper.
} 
We highlight one typical example below (for more connections, we refer to the excellent exposition by Viola [43]).

1.1.1 Connection to $\mathrm{AC}^{0}[\oplus]$-Circuits. $\mathrm{AC}^{0}[\oplus]$ denotes the class of constant-depth circuits consisting of AND, OR, XOR and NOT gates of unbounded fan-in. The celebrated polynomial approximation method, which shows lower bounds for $\mathrm{AC}^{0}[\oplus]$-circuits follow from correlation bounds against $\mathbb{F}_{2}$-polynomials, was first established by Razborov [36], who applied this connection to prove the first super-polynomial lower bounds against $\mathrm{AC}^{0}[\oplus]$, which was later refined and improved by $[39,40]$. This connection has since then become the integral tool in the study of the computational power of $\mathrm{AC}^{0}[\oplus]$-circuits, see $[8,10,26,29,34,35,45]$ for some recent work on this direction.

1.1.2 Known Progress on Correlation Bounds for Explicit Functions against $\mathbb{F}_{2}$-Polynomials. The renowned work by Razborov and Smolensky $[36,39,40]$ mentioned above proved that the majority function $\mathrm{MAJ}^{3}$ satisfies $\operatorname{corr}\left(\mathrm{MAJ}_{n}, d\right) \leq O(d / \sqrt{n})$. Although nearly 40 years passed, it remains the strongest correlation bound known for any explicit functions in NP against $\log n$-degree $\mathbb{F}_{2}$ polynomials, and no $o(1)$ correlation bounds were known for $\sqrt{n}$ degree $\mathcal{F}_{n}$-polynomials. Indeed, even the following questions are still open (see [43] for more in-depth discussions of these open problems).

Open Problem 1. Is there a function $f \in \mathrm{NP}$ such that $\operatorname{corr}(f, \log n)$ $\leq o(1 / \sqrt{n}) ?$

Open Problem 2. Is there a function $f \in \mathrm{NP}$ such that $\operatorname{corr}(f, \sqrt{n})$ $\leq o(1)$ ?

Recall that the Razborov-Smolensky bound showed that $\operatorname{corr}\left(\mathrm{MAJ}_{n}, \log n\right) \leq O(\log n / \sqrt{n})$ and $\operatorname{corr}\left(\mathrm{MAJ}_{n}, o(\sqrt{n})\right) \leq o(1){ }^{4}$ These two open questions ask to improve the Razborov-Smolensky bound for the degree $\log n$ or $\sqrt{n}$, by even a tiny amount.

For lower degrees $(d \leq o(\log n))$, stronger correlation bounds were known by $[2,6,9,18,46]$. It was also proved that very strong inverse exponential correlation bounds against constant-degree $\mathbb{F}_{2}$-polynomials would improve the state-of-the-art lower bound against unrestricted Boolean circuits [17].

1.1.3 Recent Progress on Correlation Bounds for "Semi-explicit" Functions against $\mathbb{F}_{2}$-Polynomials. Since the progress on resolving Open Problem 1 and Open Problem 2 for an explicit function in NP has been lacking for decades, it motivates the interest to exhibit a hard function in some larger complexity classes first.

In fact, Open Problem 1 was even open for the gigantic classes $\mathrm{E}^{\mathrm{NP}}$, until the recent independent work by Chen and Ren [13] and Viola [45]. In [13], a $\left(1 / 2+2^{-\operatorname{polylog}(n)}\right)$-inapproximability bound for $\mathrm{NQP}^{5}$ against $\mathrm{ACC}^{0}$ was proved, which implies that there is a function $f \in \mathrm{NQP}$ such that $\operatorname{corr}(f, \operatorname{polylog}(n)) \leq 2^{-\operatorname{polylog}(n)}$. In Viola [45], it is established that there is a function $f \in \mathrm{E}^{\mathrm{NP}}$ such that $\operatorname{corr}\left(f, n^{o(1)}\right) \leq n^{-1+\varepsilon}$ for any $\varepsilon>0$.

Later, a recent work by Chen, Lyu and Williams [12] proved that for every constant $\varepsilon>0$, there is a function $f \in \mathrm{E}^{\mathrm{NP}}$ such

\footnotetext{
${ }^{3}$ The function $M A J_{n}:\{0,1\}^{n} \rightarrow\{0,1\}$ outputs 1 if and only if there are more ones than zeros in its input.

${ }^{4}$ These are indeed tight for MAJ, see [44].

${ }^{5} \mathrm{NQP}:=\mathrm{NTIME}\left[n^{\text {polylog(n)}}\right]$ stands for nondeterministic quasi-polynomial time.
}

that $\operatorname{corr}\left(f, n^{1 / 2-\varepsilon}\right) \leq \exp \left(-n^{\Omega_{\varepsilon}(1)}\right)$, where $\Omega_{\varepsilon}(1)$ is a constant approaching 0 when $\varepsilon$ approaching 0 . However, Open Problem 2 still remained open even for $\mathrm{E}^{\mathrm{NP}}$.

\subsection{Our Results}

1.2.1 Strong Correlation Bounds "One Epsilon” Away from a Circuit Lower Bound Breakthrough. In this work, we significantly improved upon the correlation bounds from [12].

THEOREM 1.1. There is a function $f \in \mathrm{E}^{\mathrm{NP}}$ for which, for every sufficiently large $n$ and for any $d \leq o(n / \log n)^{1 / 2}$, it holds that $\operatorname{corr}\left(f_{n}, d\right) \leq 2^{-d}$.

One may wonder whether Theorem 1.1 can be further improved to, say, $\operatorname{corr}\left(f_{n}, d\right) \leq 2^{-d}$ for $d=n^{1 / 2+\varepsilon}$. We observe that such improvement will imply new depth-3 $\mathrm{AC}^{0}$-circuits lower bounds.

Theorem 1.2. For any function $d(n): \mathbb{N} \rightarrow \mathbb{N}$, if there is a function $f$ in $\mathrm{E}^{\mathrm{NP}}$ such that $\operatorname{corr}\left(f_{n}, d(n)\right) \leq 2^{-d(n)}$ for infinitely many $n \in \mathbb{N}$, then there is a function $g$ in $\mathrm{E}^{\mathrm{NP}}$ that does not admit depth-3 $\mathrm{AC}^{0}$ circuits of size at most $2^{o(d(n))}$.

Currently, the best known depth-3 $\mathrm{AC}^{0}$-circuits lower bound is $2^{\Omega(\sqrt{n})}$ [20]. It has been a notorious open question to prove better lower bounds against depth- $3 \mathrm{AC}^{0}$-circuits, even for functions in complexity class as large as $\mathrm{E}^{\mathrm{NP}}$. Therefore, improving our result, even by an "epsilon amount" on the exponent, would imply a breakthrough in constant-depth circuit lower bounds.

1.2.2 Better Degree-Error Trade-Off for $\mathrm{E}^{\mathrm{NP}}$ against $\mathbb{F}_{2}$-Polynomials. Due to some technical obstacles, the proof of Theorem 1.1 does not give any non-trivial correlation bounds for higher degrees (i.e., $d \geq \sqrt{n}$ ). Still, using a different proof, we managed to show a trade-off between error and degree for $\mathrm{E}^{\mathrm{NP}}$ against degree- $d \mathbb{F}_{2}$ polynomials.

THeOREM 1.3. For every $\beta \in(0,1)$, there is an $\mathrm{E}^{\mathrm{NP}}$ function $f$ such that, for every sufficiently large $n$, it holds that $\operatorname{corr}\left(f, n^{\beta} / \log n\right) \leq$ $\exp \left(-\Omega\left(n^{\frac{2}{3}(1-\beta)}\right)\right)$.

Setting $\beta=0.5$, Theorem 1.3 resolved Open Problem 2 for $\mathrm{E}^{\mathrm{NP}}$ in a very strong way. It gives an inverse exponential correlation of $2^{-\widetilde{\Omega}\left(n^{1 / 3}\right)}$ against $\sqrt{n}$-degree $\mathbb{F}_{2}$-polynomials. However, we remark that this does not match the correlation bound in Theorem 1.1 when $\beta$ is slightly less than 0.5 , and we leave it as an interesting open question to obtain a better trade-off. (We believe that $\operatorname{corr}(f, d) \leq$ $\exp \left(-\Omega\left(n^{\frac{2}{3}(1-\beta)} / \log n\right)\right)$ can be further improved to $\operatorname{corr}(f, d) \leq$ $\exp \left(-\Omega\left(n^{(1-\beta)} / \log n\right)\right)$, for all $\beta \in(0,1)$.)

1.2.3 $\mathrm{P}^{\mathrm{NP}}$ Construction of Extremely Rigid Matrices. In fact, the proof of Theorem 1.3 above builds on the techniques behind the $\mathrm{P}^{\mathrm{NP}}$-construction of rigid matrices [1,5]. More specifically, we rely on the rectangular PCPs constructed in [5]. Fully utilizing this technique, we can also construct extremely rigid matrices over $\mathbb{F}_{2}$ in $\mathrm{P}^{\mathrm{NP}}$.

We first recall the definition of rigid matrices.

Definition 1.4. For $r, n \in \mathbb{N}$ and a matrix $M \in \mathbb{F}_{2}^{n \times n}$, the $r$ rigidity of $M$, denoted as $\mathcal{R}_{M}(r)$, is the minimum number of entries one needs to change in $M$ to make its rank over $\mathbb{F}_{2}$ at most $r$. 
THEOREM 1.5. For every constant $\varepsilon \in(0,1)$, there is a $\mathrm{P}^{\mathrm{NP}}$ algorithm which on input $1^{n}$ outputs an $n \times n \mathbb{F}_{2}$-matrix $H_{n}$ satisfying $\mathcal{R}_{H_{n}}\left(2^{\log ^{1-\varepsilon} n}\right) \geq\left(1 / 2-\exp \left(-\log ^{2 / 3 \cdot \varepsilon} n\right)\right) \cdot n^{2}$, for every sufficiently large $n$.

The matrix $H$ constructed in Theorem 1.5 is extremely rigid in the sense that even $\mathcal{R}_{H_{n}}$ (1) cannot be greater than $1 / 2 \cdot n^{2}$ (either the all-zero or the all-one matrix agrees with $H$ on at least $1 / 2 \cdot n^{2}$ entries).

Comparison with previous work. The problem of efficiently constructing rigid matrices is a longstanding open problem in complexity theory [30, 37, 42].

Alman and Chen [1] established a PNP construction of matrices $H_{n}$ satisfying $\mathcal{R}_{H_{n}}\left(2^{\log ^{1 / 4-\varepsilon} n}\right) \geq \Omega\left(n^{2}\right)$, which was later significantly improved to $\mathcal{R}_{H_{n}}\left(2^{\log n / \Omega(\log \log n)}\right) \geq \Omega\left(n^{2}\right)$ by Bhangale, Harsha, Paradise and Tal [5]. The constructions in both of [1] and [5] are infinitely-often constructions in the sense that they only work for infinitely many input lengths $n$. [12] recently improved [5]'s construction so that it also works almost everywhere, in the sense that it now construct rigid matrices for every sufficiently large input length $n$.

Our construction improved upon all the previous work by further showing an almost-everywhere $\mathrm{P}^{\mathrm{NP}}$ construction of extremely rigid matrices.

Independent Work. Without using our new derandomized XOR lemma, it is possible to combine the known techniques in [12] and [5] in a very non-trivial way to obtain a weaker trade-off that $\mathcal{R}_{H_{n}}\left(2^{\log ^{1-\varepsilon} n}\right) \geq\left(1 / 2-\exp \left(-\log ^{1 / 2 \cdot \varepsilon} n\right)\right) \cdot n^{2}$, which would also resolve Open Problem 2 for $\mathrm{E}^{\mathrm{NP}}$. Such a weaker trade-off has been independently proved by $\mathrm{Lu}$ [31] and by Huang and Viola [23]. ${ }^{6}$

The common insight in our work and both [31] and [23] is that we can apply the classic XOR Lemma even in the setting of constructing rigid matrices.

1.2.4 Nondeterministic PRGs (NPRGs) with Near-Logarithmic SeedLength from Non-Trivial Algorithms. In [12], it was shown that for a typical circuit class $C$, a $2^{n-n^{\varepsilon}}$-time CAPP algorithm ${ }^{7}$ for $2^{n^{\varepsilon}}$ size $C$-circuits implies an infinitely-often NPRGs (i.o.-NPRGs) ${ }^{8}$ for polynomial-size $C$ with polylog $(n)$ seed length. Combing with the corresponding algorithm for polynomial-size $\mathrm{ACC}^{0}$ [48], this immediately implies an i.o.-NPRG for $\mathrm{ACC}^{0}$ with polylog $(n)$ seed length.

Naturally, one may wonder whether that seed length can be improved to the optimal $O(\log n)$ if one starts with a $2^{n-\varepsilon n}$-time algorithm instead. We show that this is the case, by proving the following theorem.

\footnotetext{
${ }^{6}$ More precisely, [23] and [31] stated a more fine-grained result that $\mathcal{R}_{H n}(\rho) \geq$ $\left(1 / 2-2^{-k}\right) \cdot n^{2}$ for $\log \rho \leq \delta \log n / k(\log \log n+k)$ for a sufficiently small $\delta>0$. Our results imply the same but for $\log \rho \leq \delta \log n / \sqrt{k}(\log \log n+k)$. See the full version for more details.

${ }^{7}$ CAPP stands for Circuit Acceptance Probability Problem, which is complete for promise-BPP and is the canonical derandomization problem. Check the full version for its definition.

${ }^{8}$ That is, one can construct a non-deterministic machine that on infinitely many $n$, the machine on input $1^{n}$ runs in non-deterministic polynomial time, and outputs either a failure symbol, or a PRG $\mathcal{G}:\{0,1\}^{\ell} \rightarrow\{0,1\}^{n}$ with $\ell$-bit seeds that fools $C$-circuits of size $n$. See the full version for a formal definition.
}

TheOREM 1.6 (INFORMAL). Let $C$ be a nice circuit class. If there is an $\varepsilon>0$ such that, the \#SAT (or CAPP) problem of C ० Junta $2^{\varepsilon n-}$ circuit of size $2^{\varepsilon n}$ can be solved in $2^{(1-\varepsilon) n}$ time, then there exists an infinitely often NPRG which takes $O\left(\log n \log \log ^{2} n\right)$ bits seeds, runs in time poly $(n)$ and fools $\mathscr{C}$-circuits of size $n$.

\subsection{Our Techniques}

Perhaps more interestingly, our new results are all proved by a new derandomized XOR lemma based on approximate linear sums, and as far as we know, this is the first application of hardness amplification in the context of constructing rigid matrices. Before formally stating and discussing our new derandomized XOR lemma, it is instructive to review the XOR lemma in [12], and why it cannot be used to prove the strong correlation bounds as in Theorem 1.1.

Notation. From now on, we will always use Boolean functions to denote a function from $\{0,1\}^{*}$ to $\{-1,1\}$, where -1 and 1 are interpreted as True and False, respectively. This choice will be particularly convenient for studying and stating correlation bounds or average-case lower bounds. For two functions $f, g:\{0,1\}^{n} \rightarrow$ $\{-1,1\}$, we will use $\langle f, g\rangle$ to denote their inner product

$$
\underset{x \in\{0,1\}^{n}}{\mathbb{E}}[f(x) \cdot g(x)] .
$$

For a collection of functions $\mathcal{F}$, we always use $\mathcal{F}_{n}$ to denote the subset of $\mathcal{F}$ consisting of $n$-bit functions from $\mathcal{F}$. We will also need to define Sum $\circ \mathcal{F}$-functions: a Sum $\circ \mathcal{F}$-function $C:\{0,1\}^{n} \rightarrow \mathbb{R}$ can be written as $C(x)=\sum_{i=1}^{\ell} \alpha_{i} \cdot C_{i}(x)$, where each $\alpha_{i}$ is a real, and each $C_{i}(x)$ is an $\mathcal{F}_{n}$-function. Here $\ell$ is called the sparsity of $C$. We also use complexity $(C)$ to denote $\max \left(\ell, \sum_{i=1}^{\ell}\left|\alpha_{i}\right|\right)$.

1.3.1 The XOR lemma in [12] and Its Disadvantage. Formally, following Levin's proof of Yao's XOR Lemma [16, 28], [12] proved the following lemma.

Lemma 1.7 ([28] AND [12, Lemma 3.8]). Let $\mathcal{F}$ be a collection of functions closed under negation and restriction. For $n \in \mathbb{N}_{\geq 1}$, $\delta, \varepsilon \in(0,1)$ and every function $f:\{0,1\}^{n} \rightarrow\{-1,1\}$, if

$$
\langle f, C\rangle<(1-\delta)
$$

for every Sum $\circ \mathcal{F}_{n}$-function $C$ such that complexity $(C) \leq 10 \cdot n / \varepsilon^{2}$ and $\|C\|_{\infty} \leq 1$, then $\left\langle f^{\oplus k}, C\right\rangle \leq(1-\delta)^{k}+\varepsilon / \delta$ for any $f \in \mathcal{F} .9$

That is, given a function $f$ which cannot be weakly approximated (say, 0.99-approximated) by Sum $\circ \mathcal{F}_{n}$-functions, one can show that $f^{\oplus k}$ is strongly average-case hard for $\mathcal{F}$. The advantage of Lemma 1.7 above over other versions of XOR lemmas [16, 24, 25, 49] is that it adds minimal computational overhead from the target class $\mathcal{F}$ to the starting class Sum $\circ \mathcal{F}$, which enables $[12,13]$ to apply Williams' algorithmic method to obtain the required hardness against Sum $\circ \mathcal{F}$ using algorithms only for $\mathcal{F}$.

Still, to obtain a $2^{-\Omega(\sqrt{n})}$ correlation bound using Lemma 1.7 with a constant $\delta$ (say, $\delta=0.01$ ), one has to set $\varepsilon \approx 2^{-\sqrt{n}}$ and $k \approx \sqrt{n}$. Applying the algorithmic method, [12] indeed managed to prove that there is an $\mathrm{E}^{\mathrm{NP}}$-computable function $f:\{0,1\}^{n} \rightarrow\{-1,1\}$ which cannot be $(1-\delta)$-approximated by linear sums of $2^{\sqrt{n}}$ many $\mathbb{F}_{2}$-polynomials of degree at most $\sqrt{n}$. Applying Lemma 1.7, one

${ }^{9}$ The function $f^{\oplus k}:\left(\{0,1\}^{n}\right)^{k} \rightarrow\{-1,1\}$ is defined as $f^{\oplus k}\left(x_{1}, \ldots, x_{k}\right)=$ $\prod_{i=1}^{k} f\left(x_{i}\right)$. 
can obtain a function $\operatorname{Amp}^{f}:=f^{\oplus k}$ such that $\operatorname{corr}\left(\mathrm{Amp}^{f}, \sqrt{n}\right) \leq$ $2^{-\Omega(\sqrt{n})}$. However, this is not enough, since Amp $f$ in fact takes $m=\Theta\left(n^{1.5}\right)$ bits of input, the correlation bounds deteriorate to $\operatorname{corr}\left(\mathrm{Amp} f, m^{1 / 3}\right) \leq 2^{-\Omega\left(m^{1 / 3}\right)}$.

1.3.2 A New Derandomized XOR Lemma. To further improve the correlation bounds in [12], we managed to prove a derandomized XOR lemma based on approximate linear sums. Roughly speaking, we construct a pseudorandom instances generator $\mathcal{G}:\{0,1\}^{m} \rightarrow$ $\{0,1\}^{n k}$ that takes a seed of length $m=\widetilde{O}(n)$, and produces $k$ instances to the function $f$. We can show that our generator $\mathcal{G}$ is pseudorandom enough to fool the proof of XOR lemma, and establish the following new derandomized XOR lemma based on approximate linear sums.

Lemma 1.8 ((INFORMAL)). Let $n \in \mathbb{N}_{\geq 1}, \varepsilon \in(0,1), k=\Theta\left(\log \varepsilon^{-1}\right)$ and $\mathcal{F}=\bigcup_{n \in \mathbb{N}_{\geq 1}} \mathcal{F}_{n}$ be a function collection satisfying some technical conditions ${ }^{10}$. There is a polynomial-time generator $\mathcal{G}:\{0,1\}^{m} \rightarrow$ $\{0,1\}^{n k}$ with $m=\widetilde{O}(n)$ such that, for every function $f:\{0,1\}^{n} \rightarrow$ $\{-1,1\}$ that cannot be weakly approximated by Sum $\circ \mathcal{F}$-functions of complexity at most $O\left(n / \varepsilon^{2}\right)$, then $\left\langle f^{\oplus k} \circ \mathcal{G}, C\right\rangle \leq \varepsilon^{\Omega(1)}$ holds for every $C \in \mathcal{F}_{m}$.

The proof of our new derandomized XOR lemma is based on a "non-Boolean" generalization of the concept of hardcore sets, which is thoroughly discussed in Section 2.1.1. Such a generalization can also be used to give a completely different and duality-based proof of Lemma 1.7.

Combing Lemma 1.8 with the algorithmic method developed in $[12-14,47]$ for proving hardness against linear sums of collection of functions which admit efficient circuit-analysis algorithms, we can then obtain our improved correlation bounds against $\mathbb{F}_{2}$ polynomials and construction of extremely rigid matrices.

\section{TECHNICAL OVERVIEW}

In this section we give an overview of the proof ideas behind our new results. In Section 2.1 we define and dicuss the key con-

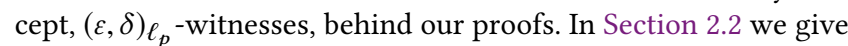
a duality-based new proof of Lemma 1.7, which can be seen as a warm-up for later proofs. In Section 2.3 we discuss the intuitions behind our proof of the new derandomization XOR lemma. In Section 2.4 we give some intuitions behind our specific constructions of restrictable generators.

Recall that we use Boolean functions to denote functions from $\{0,1\}^{*}$ to $\{-1,1\}$, where -1 and 1 are interpreted as True and False respectively. We also introduce the concept of Hölder conjugates below.

For every real $p \geq 1$, recall that the $\ell_{p}$-norm of $f$ is defined as $\|f\|_{p}:=\left(\mathbb{E}_{x \leftarrow U_{n}}|f(x)|^{p}\right)^{1 / p}$, and the $\ell_{\infty}$-norm of $f$ is defined

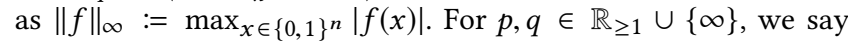
that $p$ and $q$ are Hölder conjugates of each other, if it holds that $1 / p+1 / q=1 .^{11}$

\footnotetext{
${ }^{10}$ Check the full version for the details.

${ }^{11}$ We use the convention that $1 / \infty=0$, so it can be the case that $p=1$ and $q=\infty$ and vice versa.
}

\subsection{The Dual Witness to Inapproximability by Linear Sums}

The first ingredient of our proof is a dual witness for inapproximability of a function $f$ by Sum $\circ \mathcal{F}_{n}$-functions.

Definition 2.1. Let $f:\{0,1\}^{n} \rightarrow\{-1,1\}$ be a function, let $p \in$ $\mathbb{R}_{\geq 1} \cup\{\infty\}$, and let $\delta>0, \varepsilon>0$ be two reals. We say that a function $h:\{0,1\}^{n} \rightarrow \mathbb{R}$ is $a(\delta, \varepsilon)_{\ell_{p}}$-witness for $f$ against $\mathcal{F}_{n}$-functions, if $\|h\|_{p} \leq 1,\|h\|_{1} \leq 1-\delta$ and $|\langle C, f-h\rangle| \leq \varepsilon$ for every $C \in \mathcal{F}_{n}$.

We will often consider the setting where $\varepsilon$ is very small and $\delta$ is a small constant (e.g., $\varepsilon \leq n^{-\omega(1)}$ and $\left.\delta=0.01\right)$. That is, a $(\delta, \varepsilon) \ell_{p}{ }^{-}$ witness $h$ for $f$ against $\mathcal{F}_{n}$-functions can be used to perturb $f$ so that the resulting function $f-h$ is extremely hard for $\mathcal{F}_{n}$-functions.

If additionally we can also make $1-\delta$ very small (instead of being a constant), then we would immediately obtain strong average-case lower bounds for $f$ against $\mathcal{F}_{n}$. Formally, we have the following remark.

REMARK 2.2. If there is $a(\delta, \varepsilon) \ell_{p}$-witness for $f$ against $\mathcal{F}_{n}$-functions, then $|\langle C, f\rangle| \leq \varepsilon+(1-\delta)$ for every $C \in \mathcal{F}_{n}$.

By Remark 2.2, to show that a function $f$ is strongly average-case hard against $\mathcal{F}_{n}$-functions, it suffices to construct a witness $h$ with very small $\varepsilon$ and $\ell_{1}$-norm. This will be the approach adopted in the proofs of this section.

2.1.1 $(\delta, \varepsilon) \ell_{p}$-witnesses and Hardcore Sets. One may notice that the witness defined in Definition 2.1 appears to be very similar to the concept of hardcore sets ${ }^{12}$, which are studied extensively in the complexity theory $[3,22,24,38,41]$. The following discussions in this subsection are aimed to provide more intuitions on Definition 2.1 and its connections to hardcore sets, and may be skipped without affecting the understanding of the proofs in this paper.

From $(\delta, \varepsilon)_{\ell_{\infty}}$-witnesses to (Boolean) hardcore sets. We remark quickly that when $p=\infty$, a $(\delta, \varepsilon)_{\ell_{\infty}}$-witness $h$ is essentially equivalent to an $\Omega(\delta)$-dense hardcore set of $f$. Let $P_{\text {core }}:=f-h$, since $\|h\|_{\infty} \leq 1$ and $f$ is Boolean, it immediately implies that $P_{\text {core }}(x)$ either has the same sign with $f(x)$ or is zero. We can then construct a function $f_{\text {core }}$ by setting each $f_{\text {core }}(x)=f(x)$ independently with probability $\left|P_{\text {core }}(x)\right|$, and 0 otherwise. ${ }^{13}$ Note that for every $x$, $\mathbb{E}\left[f_{\text {core }}(x)\right]=P_{\text {core }}(x)$.

We can then obtain a hardcore set of $f$ from $f_{\text {core }}$ since with high probability: (1) Since $\left\|P_{\text {core }}\right\|_{1} \geq\|f\|_{1}-\|h\|_{1} \geq \delta$, at least an $\Omega(\delta)$-fraction of $f_{\text {core }}(x)$ are non-zero (either -1 or 1$)$, those are the inputs in our hardcore set. (2) $\left\langle f_{\text {core }}, C\right\rangle$ will be very close to $\left\langle P_{\text {core }}, C\right\rangle$ (by a Chernoff bound), which is at most $\varepsilon$. This means $f$ is extremely hard on this hardcore set.

$(\delta, \varepsilon)_{\ell_{p}}$-witnesses as "non-Boolean" hardcore sets. When $p \neq \infty$, a $(\delta, \varepsilon)_{\ell_{p}}$-witness $h$ (or more accurately, the function $P_{\text {core }}^{\ell_{p}}:=f-h$ obtained by perturbing $f$ with $h$ ) can be thought of as a non-Boolean hardcore set, in the following sense: (1) $P_{\text {core }}^{\ell_{p}}$ has $\ell_{1}$-norm at least

\footnotetext{
${ }^{12}$ Roughly speaking, a hardcore set $H$ for a function $f$ is a subset of $\{0,1\}^{n}$ with at least $\delta \cdot 2^{n}$ elements such that $f$ is strongly average-case hard to compute by a certain class of functions with respect to the uniform distribution over $H$.

${ }^{13}$ If $\left\|P_{\text {core }}\right\|_{\infty}>1$, we can scale $P_{\text {core }}$ by $1 / 2$, this only reduces its density by a factor of 2 .
} 
$\|f\|_{1}-\|h\|_{1} \geq \delta$, so $P_{\text {core }}^{\ell_{p}}$ is still of " $\delta$-density" and (2) $P_{\text {core }}^{\ell_{p}}$ is still extremely hard against for $\mathcal{F}$-functions.

We cannot construct from $P_{\text {core }}^{\ell_{p}}$ a Boolean hardcore $f_{\text {core }}^{\ell_{p}}$ anymore, since many points in $P_{\text {core }}^{\ell_{p}}$ can have very large absolute values. Indeed, the norm $p$ controls the Booleanness of $f_{\text {core }}^{\ell_{p}}$ the larger the $p$ is, the closer the $f_{\text {core }}^{\ell_{p}}$ is to Boolean functions.

We also remark that another way to interpret $P_{\text {core }}^{\ell_{p}}$ is that it corresponds to a certain hardcore pseudodistribution instead of a hardcore distribution. ${ }^{14}$

2.1.2 From Inapproximability by Linear sums to $(\delta, \varepsilon)_{\ell_{p}}$-Witnesses. The following "inapproximability-to-witness" lemma shows that we can construct a non-trivial witness for $f$ against $\mathcal{F}_{n}$-function from the weak inapproximability of $f$ by Sum $\circ \mathcal{F}_{n}$-function. This lemma serves as the starting point for our derandomized XOR lemma. It can be shown by the duality of linear programming (a.k.a. the minimax theorem). We refer to the full version for its proof.

Lemma 2.3. Let $n \in \mathbb{N}_{\geq 1}$, and let $\mathcal{F}_{n}$ be a collection of $n$-input functions that is closed under negation. Let $p, q \in \mathbb{R}_{\geq 1} \cup\{\infty\}$ be such that $p$ and $q$ are Hölder conjugates of each other. For every function $f:\{0,1\}^{n} \rightarrow\{-1,1\}$ and $\delta, \varepsilon>0$, if we have

$$
\langle f, C\rangle<(1-\delta)
$$

for every Sum $\circ \mathcal{F}_{n}$-function $C$ such that complexity $(C) \leq 10 \cdot n / \varepsilon^{2}$ and $\|C\|_{q} \leq 1$, then there is a $(\delta, \varepsilon)_{\ell_{p}}$-witness $h$ for $f$ against $\mathcal{F}_{n^{-}}$ functions.

Moreover, for the case $p=\infty$ and $q=1$, the condition can be replaced by that for every $\mathrm{MAJ} \circ \mathcal{F}$-function $C$ with top-sparsity bounded by $10 n / \varepsilon^{2}$, it holds that $\langle f, C\rangle<1-2 \delta$.

REMARK 2.4. By the discussions in Section 2.1.1, when $(p, q)=$ $(\infty, 1), a(\delta, \varepsilon)_{\ell_{p}}$-witness immediately implies the existence of an $\Omega(\delta)$-dense hardcore set of $f$ against $\mathcal{F}_{n}$-functions. Thus, the moreover part of Lemma 2.3 is equivalent to Impagliazzo's Hardcore Lemma.

\subsection{A New Proof of the Original XOR Lemma}

As a warm-up, in this section we will first give a new proof of Levin's XOR Lemma [28], reformulated by [12, Lemma 3.8].

Reminder of Lemma 1.7. Let $\mathcal{F}$ be a collection of functions closed under negation and restriction. For $n \in \mathbb{N}_{\geq 1}, \delta, \varepsilon \in(0,1)$ and every function $f:\{0,1\}^{n} \rightarrow\{-1,1\}$, if

$$
\langle f, C\rangle<(1-\delta)
$$

for every Sum $\circ \mathcal{F}_{n}$-function $C$ such that complexity $(C) \leq 10 \cdot n / \varepsilon^{2}$ and $\|C\|_{\infty} \leq 1$, then $\left\langle f^{\oplus k}, C\right\rangle \leq(1-\delta)^{k}+\varepsilon / \delta$ for any $f \in \mathcal{F}$.

$\varepsilon$-Indistinguishability. For two functions $f, g:\{0,1\}^{n} \rightarrow \mathbb{R}$ and a parameter $\varepsilon>0$, we say that $f$ and $g$ are $\varepsilon$-indistinguishable by $\mathcal{F}_{n}$-functions if $|\langle f-g, C\rangle| \leq \varepsilon$ for every $C \in \mathcal{F}_{n}$.

Proof of Lemma 1.7. Applying Lemma 2.3 with $(p, q)=(1, \infty)$, the condition in the lemma implies that there is a $(\delta, \varepsilon)_{\ell_{1}}$-witness $h$ for $f$ against $\mathcal{F}_{n}$-functions. That is:

(1) $f$ and $h$ are $\varepsilon$-indistinguishable by $\mathcal{F}$-functions.

${ }^{14}$ see $[7,11]$ for more discussions on recent works in derandomization using pseudodistributions.
(2) $h$ has $\ell_{1}$-norm at most $(1-\delta)$, which is slightly less than 1 .

Proof plan. Our proof will be duality-based. That is, to show $f^{\oplus k}(x)$ is strongly average-case hard, we will show that the function $h^{\oplus k}$ is a sufficient witness to apply Remark 2.2. That is, we want to show the following:

(1) (Indistinguishability.) $f^{\oplus k}$ is $(\varepsilon / \delta)$-indistinguishable from $h^{\oplus k}$ by $\mathcal{F}$-functions.

(2) (Bounded $\ell_{1}$-norm.) $h^{\oplus k}$ has $\ell_{1}$-norm bounded by $(1-\delta)^{k}$.

The second item above is easy to establish, since $\left\|h^{\oplus k}\right\|_{1}=$ $\|h\|_{1}^{k} \leq(1-\delta)^{k}$. Hence it only remains to show the first item.

A hybrid argument. We will show the indistinguishability between $f^{\oplus k}$ and $h^{\oplus k}$ by a hybrid argument. For every $i \in\{0, \ldots, k\}$, we define a hybrid function $H_{i}^{h, f}:=h^{\oplus i} \otimes f^{\oplus k-i}$. That is, for every $r=\left(r_{1}, \ldots, r_{k}\right) \in\left(\{0,1\}^{n}\right)^{k}$, we have

$$
H_{i}^{h, f}(r)=\prod_{j=1}^{i} h\left(r_{j}\right) \cdot \prod_{j=i+1}^{k} f\left(r_{j}\right) .
$$

Note that $H_{0}^{h, f}$ and $H_{k}^{h, f}$ are just $f^{\oplus k}$ and $h^{\oplus k}$, respectively. We will show that $H_{0}^{h, f}$ and $H_{k}^{h, f}$ are indistinguishable by showing that for every $i \in\{0, \ldots, k-1\}$, the two consecutive functions $H_{i}^{h, f}$ and $H_{i+1}^{h, f}$ are indistinguishable. Formally, we have the following claim.

ClaIm 2.5. For every $i \in[k]$ and every $C \in \mathcal{F}_{n k}, \mid\left\langle H_{i-1}^{h, f}-\right.$ $\left.H_{i}^{h, f}, C\right\rangle \mid \leq \varepsilon \cdot(1-\delta)^{i-1}$.

We will prove Claim 2.5 later, but assuming it for now, for every $C \in \mathcal{F}_{n k}$, we have

$$
\begin{aligned}
\left|\left\langle f^{\oplus k}, C\right\rangle\right| & \leq\left|\left\langle h^{\oplus k}, C\right\rangle\right|+\left|\left\langle f^{\oplus k}-h^{\oplus k}, C\right\rangle\right| \\
& \leq\|h\|_{1}^{k}+\sum_{i=1}^{k}\left|\left\langle H_{i-1}^{h, f}-H_{i}^{h, f}, C\right\rangle\right| \quad\left(\|C\|_{\infty}=1\right) \\
& \leq(1-\delta)^{k}+\varepsilon \cdot \sum_{i=0}^{k-1}(1-\delta)^{i}
\end{aligned}
$$

(Claim 2.5 and $\|h\|_{1} \leq 1-\delta$ )

$$
\leq(1-\delta)^{k}+\varepsilon / \delta
$$

Finally, we prove Claim 2.5. have

Proof of Claim 2.5. From the definition of $H_{i-1}^{h, f}$ and $H_{i}^{h, f}$, we

$$
\begin{aligned}
& \left\langle H_{i-1}^{h, f}-H_{i}^{h, f}, C\right\rangle= \\
& \underset{r \leftarrow U_{n k}}{\mathbb{E}}\left[C\left(r_{1}, \ldots, r_{k}\right) \cdot \prod_{j=1}^{i-1} h\left(r_{j}\right) \cdot \prod_{j=i}^{k} f\left(r_{j}\right)\right]- \\
& \underset{r \leftarrow U_{n k}}{\mathbb{E}}\left[C\left(r_{1}, \ldots, r_{k}\right) \cdot \prod_{j=1}^{i} h\left(r_{j}\right) \cdot \prod_{j=i+1}^{k} f\left(r_{j}\right)\right] .
\end{aligned}
$$

We use $r_{-i}$ to denote $\left(r_{1}, \ldots, r_{i-1}, r_{i+1}, \ldots, r_{k}\right) \in\left(\{0,1\}^{n}\right)^{k-1}$, so that $r \in\{0,1\}^{n k}$ can be decomposed into $r_{i}$ and $r_{-i}$. Organizing 
the right side of (2.2), we have

$$
\begin{aligned}
& \left\langle H_{i-1}^{h, f}-H_{i}^{h, f}, C\right\rangle= \\
& \underset{r_{-i} \leftarrow U_{n(k-1)}}{\mathbb{E}} \prod_{j=1}^{i-1} h\left(r_{j}\right) \prod_{j=i+1}^{k} f\left(r_{j}\right) \underset{r_{i} \leftarrow U_{n}}{\mathbb{E}}\left[C\left(r_{1}, \ldots, r_{k}\right) \cdot\left(f_{i}\left(r_{i}\right)-h_{i}\left(r_{i}\right)\right)\right] .
\end{aligned}
$$

To further bound (1), for each $r_{-i} \in\left(\{0,1\}^{n}\right)^{k-1}$, we define a function $D^{r_{-i}}:\{0,1\}^{n} \rightarrow\{-1,1\}$ as

$$
D^{r_{-i}}(x):=C\left(r_{1}, \ldots, r_{i-1}, x, r_{i+1}, \ldots, r_{k}\right) .
$$

It follows that $D^{r_{-i}} \in \mathcal{F}_{n}$ since $\mathcal{F}$ is closed under restriction. Therefore, since $h$ is a $(\delta, \varepsilon)_{\ell_{1}}$-witness for $f$ against $\mathcal{F}_{n}$-functions, we have

$$
\left|\left\langle f-h, D^{r_{-i}}\right\rangle\right| \leq \varepsilon
$$

Plugging in (1), we have

$$
\begin{aligned}
\left|\left\langle H_{i-1}^{h, f}-H_{i}^{h, f}, C\right\rangle\right| & =\left|\underset{r_{-i} \leftarrow U_{n(k-1)}}{\mathbb{E}} \prod_{j=1}^{i-1} h\left(r_{j}\right) \prod_{j=i+1}^{k} f\left(r_{j}\right)\left\langle f-h, D^{r_{-i}}\right\rangle\right| \\
& \leq \varepsilon \cdot \underset{r_{-i} \leftarrow U_{n(k-1)}}{\mathbb{E}} \prod_{j=1}^{i-1}\left|h\left(r_{j}\right)\right| \\
& \leq \varepsilon \cdot \prod_{j=1}^{i-1} \underset{r_{j} \leftarrow U_{n}}{\mathbb{E}}\left|h\left(r_{j}\right)\right| \\
& \leq \varepsilon \cdot(1-\delta)^{i-1} .
\end{aligned}
$$

\subsection{New Derandomized XOR Lemma}

Now we turn to the proof intuitions behind the proof of our new derandomized XOR lemma. We begin by introducing the concept of pseudorandom instance generator and some useful notation.

Pseudorandom instance generators and notation. For convenience, let $\mathcal{F}=\bigcup_{n \in \mathbb{N} \geq 1} \mathcal{F}_{n}$ be a collection of functions closed under negation and restriction. We will always use $f:\{0,1\}^{n} \rightarrow\{-1,1\}$ to denote a weakly average-case hard function on which we will apply the hardness amplification, and we will always use $n$ to denote the input length of $f$.

The idea is to use a pseudorandom instance generator $\mathcal{G}:\{0,1\}^{m} \rightarrow$ $\left(\{0,1\}^{n}\right)^{k}$ ( $m$ is much less than $\left.n k\right)$ to generate inputs to the function $f^{\oplus k}$, similar to the original derandomized XOR lemma in [25]. That is, by directly composing the generator $\mathcal{G}$ and $f^{\oplus k}$, one obtains a function $\operatorname{Amp}^{f}:=f^{\oplus k} \circ \mathcal{G}$, which has input length $m$ instead of $n k$.

High-level idea. Our goal would be to construct the desired pseudorandom instance generator $\mathcal{G}$ such that a similar argument as in the proof of Lemma 1.7 still goes through. That is, we wish to show that $\mathrm{Amp}^{h}:=h^{\oplus k} \circ \mathcal{G}$ is a dual-witness showing that $\operatorname{Amp}^{f}=f^{\oplus k} \circ \mathcal{G}$ is strongly average-case hard against $\mathcal{F}$-functions (see Remark 2.2). Therefore, we need to establish the following two statements:
(1) (Indistinguishability.) $\mathrm{Amp}^{f}$ and $\mathrm{Amp}^{h}$ are $\left(\varepsilon^{\Omega(1)}\right)$-indistinguishable by $\mathcal{F}_{m}$-functions.

(2) (Bounded $\ell_{1}$-norm.) Amp ${ }^{h}$ has $\ell_{1}$-norm at most $(1-\delta)^{k}$.

In the following, we show how to construct a generator meeting the two requirements above. We will omit some technical details and focus on the key insights in our approach.

2.3.1 Establishing the Indistinguishability. First, our constructed $\mathcal{G}$ needs to ensure that Amp $f=f^{\oplus k} \circ \mathcal{G}$ and Amp ${ }^{h}=h^{\oplus k} \circ \mathcal{G}$ are indistinguishable. In the following we will try to adapt the proof of Lemma 1.7, and figure out along the way that which properties $\mathcal{G}$ has to satisfy for the adaption to go through.

A new hybrid argument and the difficulty. Again we will try to apply a hybrid argument, recall that we have defined the hybrid functions $H_{i}^{h, f}=h^{\oplus i} \otimes f^{\oplus k-i}$ in the proof of Lemma 1.7. To simplify notation, we let $\mathcal{G}_{i}^{h, f}=H_{i}^{h, f} \circ \mathcal{G}$ to denote our new hybrid functions. Note that $\mathcal{G}_{0}^{h, f}=\operatorname{Amp}^{f}$ and $\mathcal{G}_{k}^{h, f}=\mathrm{Amp}^{h}$.

Fix $i \in[k]$, our goal is to show that $\left|\left\langle\mathcal{G}_{i-1}^{h, f}-\mathcal{G}_{i}^{h, f}, C\right\rangle\right|$ is small for every $C \in \mathcal{F}_{m}$. Recall in the proof of Lemma 1.7, an analogous bound (Claim 2.5) is proved by considering the following equalities:

$$
\begin{aligned}
& \left|\left\langle H_{i-1}^{h, f}-H_{i}^{h, f}, C\right\rangle\right| \\
& =\left|\underset{r \leftarrow U_{n k}}{\mathbb{E}} \prod_{j=1}^{i-1} h\left(r_{j}\right) \cdot \prod_{j=i+1}^{k} f\left(r_{j}\right) \cdot\left[C\left(r_{1}, \ldots, r_{k}\right) \cdot\left(f_{i}\left(r_{i}\right)-h_{i}\left(r_{i}\right)\right)\right]\right| \\
& =\left|\underset{r_{-i} \leftarrow U_{n(k-1)}}{\mathbb{E}} \prod_{j=1}^{i-1} h\left(r_{j}\right) \cdot \prod_{j=i+1}^{k} f\left(r_{j}\right) \cdot\left\langle f-h, D^{r_{-i}}\right\rangle\right|,
\end{aligned}
$$

where $D^{r_{-i}}:\{0,1\}^{n} \rightarrow\{-1,1\}$ is obtained by restricting the inputs $r_{1}, \ldots, r_{i-1}, \ldots, r_{i+1}, \ldots, r_{k}$ to $C$ by $r_{-i}$. Since $\mathcal{F}$ is closed under restriction, it follows that $D^{r_{i}} \in \mathcal{F}$, and we can then apply the bound on $\left|\left\langle f-h, D^{r_{-i}}\right\rangle\right|$, since $f$ and $h$ are indistinguishable by $\mathcal{F}_{n}$-functions.

The key intuition in the proof above is that, since the $i$-th input $r_{i}$ in $r=\left(r_{1}, \ldots, r_{k}\right)$ is completely independent to the other $k-1$ inputs, one can fix the other inputs first and then apply the indistinguishability between $f$ and $h$ to replace $f$ by $h$ on $r_{i}$.

Switching to our new setting. For a seed $r \in\{0,1\}^{m}$, we use $\tilde{r}_{i}$ to denote $\mathcal{G}(r)_{i}$ for simplicity. Then we can still write

$$
\begin{aligned}
& \left|\left\langle\mathcal{G}_{i-1}^{h, f}-\mathcal{G}_{i}^{h, f}, C\right\rangle\right| \\
= & \underset{r \leftarrow U_{m}}{\mathbb{E}} \prod_{j=1}^{i-1} h\left(\tilde{r}_{j}\right) \cdot \prod_{j=i+1}^{k} f\left(\tilde{r}_{j}\right) \cdot\left[C(r) \cdot\left(f_{i}\left(\tilde{r}_{i}\right)-h_{i}\left(\tilde{r}_{i}\right)\right)\right] .
\end{aligned}
$$

But since now all the $\tilde{r}_{i}$ are no longer independent (since they are generated from a seed $r$ with length $m$ much less than $n k$ ). We cannot proceed as (5) anymore. That is, if we try to fix $\tilde{r}_{-i}$ first, then it may even completely fix the value of $\tilde{r}_{i}$, and we can no longer obtain a similar function $D^{\tilde{r}_{-i}}$ on $\tilde{r}_{i}$.

Partial independence and $\mathcal{F}$-restrictable generators. Inspired by the famous Nisan-wigderson generator [33], and similar to the proof of the original derandomized XOR lemma in [25]. Our idea to resolve the issue above is to design the generator $\mathcal{G}$ in a way that, 
for each $i$, some part of the seed $r$ directly corresponds to $\tilde{r}_{i}$, yet for all other bits, they are almost independent to $\tilde{r}_{i}$.

More formally, we want a mapping $T_{i}:\{0,1\}^{n} \times\{0,1\}^{m-n} \rightarrow$ $\{0,1\}^{m}$, such that: (1) $T_{i}$ is a bijection and (2) $\mathcal{G}\left(T_{i}(x, \alpha)\right)_{i}=x$ for all $(x, \alpha) \in\{0,1\}^{n} \times\{0,1\}^{m-n}$. That is, the first condition says that $T_{i}$ is just a "reorganization" of the input space $\{0,1\}^{m}$ while the second condition says that $x$ corresponds directly to $\tilde{r}_{i}$.

Using the mapping $T_{i}$, we can write

$$
\begin{aligned}
& \left|\left\langle\mathcal{G}_{i-1}^{h, f}-\mathcal{G}_{i}^{h, f}, C\right\rangle\right|= \\
& \mid \underset{\substack{(x, \alpha) \leftarrow U_{m} \\
r=T_{i}(x, \alpha)}}{\mathbb{E}} \prod_{j=1}^{i-1} h\left(\tilde{r}_{j}\right) \cdot \prod_{j=i+1}^{k} f\left(\tilde{r}_{j}\right) \cdot\left[C(r) \cdot\left(f_{i}(x)-h_{i}(x)\right)\right]
\end{aligned}
$$

For $\alpha \in\{0,1\}^{m-n}$ and $x \in\{0,1\}^{n}$, we let

$$
D^{\alpha}(x):=\prod_{j=1}^{i-1} h\left(\tilde{r}_{j}\right) \cdot \prod_{j=i+1}^{k} f\left(\tilde{r}_{j}\right) \cdot C\left(T_{i}(x, \alpha)\right),
$$

where the $\tilde{r}_{j}$ above corresponds to $\mathcal{G}\left(T_{i}(x, \alpha)\right)_{j}\left(\mathcal{G}(r)_{j}\right.$ if $\left.r=T_{i}(x, \alpha)\right)$.

Plugging in the above into (2.3.1), it follows that

$$
\left|\left\langle\mathcal{G}_{i-1}^{h, f}-\mathcal{G}_{i}^{h, f}, C\right\rangle\right|=\underset{\alpha \leftarrow U_{m-n}}{\mathbb{E}}\left\langle f-h, D^{\alpha}\right\rangle .
$$

Therefore, if the function $D^{\alpha}$ above still belongs to $\mathcal{F}_{n}$, we can then apply the indistinguishability between $f$ and $h$ by $\mathcal{F}_{n^{-}}$ functions, and proceed just as in the proof of Lemma 1.7. This motivates our definition of $\mathcal{F}$-restrictable generator as follows.

Definition 2.6 ( $\mathcal{F}$-Restrictable Generators). Given a function collection $\mathcal{F}$ and $n \in \mathbb{N}_{\geq 1}$, a generator $\mathcal{G}:\{0,1\}^{m} \rightarrow\{0,1\}^{n k}$ is called $\mathcal{F}$-restrictable, if there are $k$ embedding functions $T_{1}, \ldots, T_{k}$ : $\{0,1\}^{n} \times\{0,1\}^{m-n} \rightarrow\{0,1\}^{m}$ such that the following hold:

(1) All the $T_{i}$ are bijections.

(2) For everyi $\in[k]$ and $(x, \alpha) \in\{0,1\}^{n} \times\{0,1\}^{m-n}, \mathcal{G}\left(T_{i}(x, \alpha)\right)_{i}$ $=x$. That is, $T_{i}(x, \alpha) \in\{0,1\}^{m}$ is a seed to $\mathcal{G}$ which fixes the $i$-th instance of $\mathcal{G}\left(T_{i}(x, \alpha)\right)$ to be $x$.

(3) For every $\mathcal{F}_{m}$-function $C:\{0,1\}^{m} \rightarrow\{-1,1\}, i \in[k], \alpha \in$ $\{0,1\}^{m-n}$ and functions $u_{1}, \ldots, u_{k}:\{0,1\}^{n} \rightarrow\{1,-1\}$, the function $D(x):=C\left(T_{i}(x, \alpha)\right) \cdot \prod_{j \in[k] \backslash\{i\}} u_{j}\left(\mathcal{G}\left(T_{i}(x, \alpha)\right)_{j}\right) b e-$ longs to $\mathcal{F}_{n}$.

In the proof of the original derandomized XOR Lemma by [25], a restrictable generator for small circuits was constructed by directly adapting the Nisan-Wigderson generator [33], which is unfortunately not enough for our applications. So instead, we design two restrictable generators which are tailored to $\mathbb{F}_{2}$-polynomials and low-rank matrices.

To prove Theorem 1.1, we carefully construct a "star-like" $\mathcal{F}$. restrictable generator for a function collection $\mathcal{F}$ which contains low-degree polynomials as a subset. And similarly, we design a "bicoloring" restrictable generator for low-rank matrices to prove Theorem 1.5. We will overview the high-level ideas behind these constructions in Section 2.4.

If the function $h$ is Boolean as well, then Item (3) of Definition 2.6 tells us $D^{\alpha} \in \mathcal{F}_{n}$ and we can then bound (2). However, the function $h$ may be non-Boolean, and in fact, it may be even unbounded. This causes $D^{\alpha}$ to also be a non-Boolean function, and we can not directly apply the third condition in Definition 2.6.
Smooth witnesses come to help. We first observe that the aforementioned issue can be resolved if $h$ is smooth in a certain sense. Suppose $h$ is $[-1,1]$-valued (that is, $\left.\|h\|_{\infty} \leq 1\right)$, we can view $D^{\alpha}(x)$ as a probabilistic $\mathcal{F}$-function and apply a similar argument.

In more details, we sample $i-1$ independent functions $u_{1} \ldots u_{i-1}$ mapping $\{0,1\}^{n}$ to $\{0,1\}$ from certain distributions, in a way that for every $x \in\{0,1\}^{n}$, letting $r=T_{i}(x, \alpha)$, we have

$$
\underset{u_{j}}{\mathbb{E}}\left[u_{j}\left(\tilde{r}_{j}\right)\right]=h\left(\tilde{r}_{j}\right) \quad \text { for every } j \in[i-1] \text {. }
$$

We then set

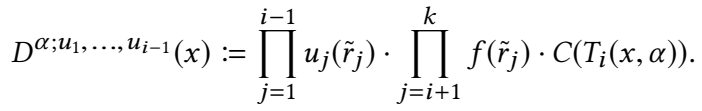

Recall that we have set $r=T_{i}(x, \alpha)$, and hence $\tilde{r}_{j}$ above corresponds to $\mathcal{G}\left(T_{i}(x, \alpha)\right)_{j}$.

By Item (3) of Definition 2.6, $D^{\alpha ; u_{1}, \ldots, u_{i-1}}$ is an $\mathcal{F}_{n}$-function for every possible $(i-1)$-tuples $\left(u_{1}, \ldots, u_{i-1}\right)$. Hence, we have

$$
\begin{aligned}
\left|\left\langle\mathcal{G}_{i-1}^{h, f}-\mathcal{G}_{i}^{h, f}, C\right\rangle\right| & =\underset{\alpha \leftarrow U_{m-n}}{\mathbb{E}}\left\langle f-h, D^{\alpha}\right\rangle . \\
& =\underset{\alpha \leftarrow U_{m-n}}{\mathbb{E}} \underset{u_{1} \ldots u_{i-1}}{\mathbb{E}}\left\langle f-h, D^{\left.\alpha ; u_{1} \ldots u_{i-1}\right\rangle}\right. \\
& \leq \varepsilon .
\end{aligned}
$$

When $\|h\|_{\infty} \leq M$, a simple scaling argument (replace $h$ by $h / M$ ) can be used to show that $\left|\left\langle\mathcal{G}_{i-1}^{h, f}-\mathcal{G}_{i}^{h, f}, C\right\rangle\right| \leq M^{i-1} \cdot \varepsilon$.

Norms and Smoothness of the witnesses. Setting $(p, q)=(\infty, 1)$, Lemma 2.3 shows that if we can show that $f$ is weakly inapproximable by Sum $\circ \mathcal{F}_{n}$-functions of unit $\ell_{1}$-norm, then we would obtain a $(\delta, \varepsilon)_{\ell_{\infty}}$-witness $h$. And one can then proceed to prove our derandomized XOR lemma.

Unfortunately, due to some inherent limitation of the polynomial method, using the algorithmic method, it seems very hard to prove there is a function $f \in \mathrm{E}^{\mathrm{NP}}$ which is weakly inapproximable by Sum $\circ \mathcal{F}_{n}$-functions of unit $\ell_{1}$-norm. ${ }^{15}$

By carefully analyzing the approaches in [12-14], we adapt the algorithmic method to show that $f$ is weakly inapproximable by Sum $\circ \mathcal{F}_{n}$-functions (think of $\mathcal{F}_{n}$ as low-degree $\mathbb{F}_{2}$-polynomials) of unit $\ell_{4}$-norm. By Lemma 2.3, this gives us a $(\delta, \varepsilon)_{\ell_{4 / 3}}$-witness $h$.

The bound on the $\ell_{4 / 3}$-norm of $h$ imposes a smoothness condition on $h$. Formally, since $\mathbb{E}_{x \leftarrow U_{n}}\left[|h(x)|^{4 / 3}\right] \leq 1$, for every $t \geq 1$, it holds that

$$
\underset{x \leftarrow U_{n}}{\mathbb{E}}\left[|h(x)| \cdot 1_{h(x) \mid \geq t}\right] \leq t^{-1 / 3} \underset{x \leftarrow U_{n}}{\mathbb{E}}\left[|h(x)|^{4 / 3}\right] \leq t^{-1 / 3} .
$$

That is, the total mass of "heavy points" in $h$ is very small. This inspires us to decompose the $h$ as the sum of two functions $h_{\text {light }}$ and $h_{\text {heavy }}$, where $h_{\text {light }}(x):=h(x) \cdot 1_{|h(x)|<t}$ and $h_{\text {heavy }}(x):=$ $h(x) \cdot \mathbf{1}_{|h(x)| \geq t}$. Now, since $\left\|h_{\text {light }}\right\|_{\infty}$ is a most $t$, one can deal with it with the approach discussed above. For $h_{\text {heavy }}$, since $\left\|h_{\text {heavy }}\right\|_{1}$ is small, one may try to simply ignore this part.

\footnotetext{
${ }^{15}$ Indeed, this is impossible if we relax the condition on the total sum of absolute values of coefficients in $C \in$ Sum $\circ \mathcal{F}_{n}$ : Fixing a function $f:\{0,1\}^{n} \rightarrow\{-1,1\}$, one can always construct a Sum $\circ \mathcal{F}_{n}$-function $C$ such that $C\left(0^{n}\right)=2^{n} \cdot f\left(0^{n}\right)$ while $C(x)=0$ for every $x \neq 0^{n}$, by summing only two functions. This $C$ is of sparsity 2 , and satisfies $\|C\|_{1}=1$ and $\langle C, f\rangle=2^{-n} \cdot 2^{n}=1$. On the other hands, the algorithmic method can still be used to show weak-inapproximability by Sum $\circ \mathcal{F}_{n}$ with unit $\ell_{4}$-norm, even allowing the coefficients to be $2^{O(n)}$.
} 
The real execution of the above plan is, however, much more complicated than the above sounds. For one, after decomposing $h$ into 2 parts, the function

$$
D^{\alpha}(x):=\prod_{j=1}^{i-1} h\left(\tilde{r}_{j}\right) \cdot \prod_{j=i+1}^{k} f\left(\tilde{r}_{j}\right) \cdot C\left(T_{i}(x, \alpha)\right)
$$

actually breaks into $2^{i-1}$ parts. We have to carefully make sure that this exponential blow-up does not cancel any advantage we gain in the decomposition.

Indeed, a simple two-way decomposition seems not sufficient, and in the real proof (which can be found in the full version), we will actually decompose $h$ into many levels, where the $k$-th level is defined as $h_{k}(x):=h(x) \cdot \mathbf{1}_{|h(x)| \in\left(2^{k-1}, 2^{k}\right]}$ (together with $h_{0}(x):=$ $\left.h(x) \cdot \mathbf{1}_{|h(x)| \leq 1}\right)$, and apply a novel way to partition the exponential parts of $D^{\alpha}\left(\tilde{r}_{i}\right)$ into only polynomially many groups, and bound each of them separately. Our decomposition is somewhat similar to the analysis of the "bounded independence plus noise" framework for constructing PRGs developed by Haramaty, Lee, and Viola in [19, 27], which is later used by Forbes and Kelley to construct PRGs for unordered branching programs [15]. ${ }^{16}$

2.3.2 Bounding the $\ell_{1}$-Norm. Finally, let us turn to the second condition we wish to establish for $\mathrm{Amp}^{h}$, which requires us to bound its $\ell_{1}$-norm.

Mixing $\mathrm{Amp}^{h}$ by introducing fresh randomness. Since we essentially have no control over $\mathrm{Amp}^{h}$, if the generator $\mathcal{G}$ always "hits" the parts of $h$ with large magnitude, then $\mathrm{Amp}^{h}$ could have $\ell_{1}$-norm even larger than 1. For example, if for all $r \in\{0,1\}^{m}$ and $i \in[k]$ it holds $h\left(\tilde{r}_{i}\right) \geq 1$, then clearly $\left\|\mathrm{Amp}^{h}\right\|_{1} \geq 1$ as well.

We resolve this issue by introducing some new fresh randomness to "mix" different parts of $h$. To see the idea, let $\mathcal{G}$ be an arbitrary generator. Suppose that we sample $k$ uniformly random strings from $\{0,1\}^{n}$, denoted by $w=\left(w_{1}, \ldots, w_{k}\right) \in\left(\{0,1\}^{n}\right)^{k}$. We then consider the following generator

$$
\mathcal{G}^{w}(r):=\left(\mathcal{G}(r)_{1} \oplus w_{1}, \ldots, \mathcal{G}(r)_{k} \oplus w_{k}\right) .
$$

We can similarly define

$$
\mathrm{Amp}^{f ; w}:=f^{\oplus k} \circ \mathcal{G}^{w} \text { and } \mathrm{Amp}^{h ; w}:=h^{\oplus k} \circ \mathcal{G}^{w} .
$$

For any fixed $r \in\{0,1\}^{m}$, we have

$$
\begin{aligned}
\underset{\left(w_{1}, \ldots, w_{k}\right) \leftarrow U_{n k}}{\mathbb{E}}\left|\operatorname{Amp}^{h ; w}(r)\right| & =\underset{\left(w_{1}, \ldots, w_{k}\right) \leftarrow U_{n k}}{\mathbb{E}} \prod_{i=1}^{k} h\left(\mathcal{G}(r)_{i} \oplus w_{i}\right) \\
& =\|h\|_{1}^{k} \leq(1-\delta)^{k} .
\end{aligned}
$$

The second equality above holds since all the $w_{i}$ are i.i.d., which means the strings $\left\{\mathcal{G}(r)_{i} \oplus w_{i}\right\}_{i \in[k]}$ are i.i.d. as well.

Hence, taking an average over all $r \in\{0,1\}^{m}$, we have

$$
\begin{aligned}
\underset{\left(w_{1}, \ldots, w_{k}\right) \leftarrow U_{n k}}{\mathbb{E}}\left\|\mathrm{Amp}^{h ; w}\right\|_{1} & =\underset{\left(w_{1}, \ldots, w_{k}\right) \leftarrow U_{n k}}{\mathbb{E} r \leftarrow U_{m}} \underset{\mathbb{E}}{\mathbb{E}}\left|\mathrm{Amp}^{h ; w}(r)\right| \\
& \leq(1-\delta)^{k} .
\end{aligned}
$$

\footnotetext{
${ }^{16}$ In more details, in the analysis of [15], they partition all monomials of a polynomial into roughly $n$ groups depending on when the monomials become "heavy" (see $[15$ Proposition 6.1]). For the exponentially many terms resulting from decomposing $h$, we also partition them into roughly $n$ groups depending on when they become "heavy". The definitions of "heavy" in our work and [15] differ since we are in very different settings.
}

With some complications, we will still be able to show that $\mathrm{Amp}^{h ; w}$ and Amp ${ }^{f ; w}$ are indistinguishable. ${ }^{17}$ This is not surprising at all: for every fixed $w=\left(w_{1}, \ldots, w_{k}\right)$ the overall effect of $w$ to the generator is simply flipping some input bits to the functions $f$ and $h$.

Full derandomization by PRGs for space-bounded computation. However, sampling $w$ still requires $n k$ bits, so it may seem we did not gain anything. Our final proof ingredient is to show that we can in fact generate "good enough" $w$ by PRGs for space-bounded computation ([32]), which only require seeds of length $O(n \log k)$. We denote this generator as $\mathcal{G}_{\text {Nisan }}:\{0,1\}^{O(n \log k)} \rightarrow\{0,1\}^{n k}$, and take our final generator $\mathcal{G}_{\text {final }}$ as $\mathcal{G}_{\text {final }}\left(r_{1}, r_{2}\right):=\mathcal{G}\left(r_{1}\right) \oplus \mathcal{G}_{\text {Nisan }}\left(r_{2}\right)$, where $\oplus$ denotes the bit-wise XOR. We remark that PRGs for spacebounded computation is also used in the proof of hardness amplification for NP [21, Lemma 5.7], although the usage there is quite different from our usage.

\subsection{Specific Restriction Generators for $\mathbb{F}_{2}$-Polynomials and Low-Rank Matrices}

In this subsection, we give a high-level overview of our restrictable generators for $\mathbb{F}_{2}$-Polynomials and low-rank matrices. Recall the definition of a $\mathcal{F}$-restrictable generator $\mathcal{G}:\{0,1\}^{m} \rightarrow\{0,1\}^{n k}$ : for every $i \in[k]$, advice $\alpha \in\{0,1\}^{m-n}$, functions $u_{1}, u_{2}, \ldots, u_{k}:\{0,1\}^{n}$ $\rightarrow\{-1,1\}$ and $C \in \mathcal{F}_{m}$, the function:

$$
D(x):=C\left(T_{i}(x, \alpha)\right) \cdot \prod_{j \in[k] \backslash\{i\}} u_{j}\left(\mathcal{G}\left(T_{i}(x, \alpha)\right)_{j}\right) .
$$

is an $\mathcal{F}_{n}$-function.

2.4.1 The Star-Like Generator for Correlation Bounds. Roughly speaking, the smaller the function class $\mathcal{F}$, the harder it is to get a restrictable generator for $\mathcal{F}$. Since low-degree $\mathbb{F}_{2}$-polynomials are not very expressive, it seems extremely hard to obtain a restrictable generator for them directly. On the other hand, exactly due to the fact that low-degree $\mathbb{F}_{2}$-polynomials are simple enough to be analyzed non-trivially by algorithms, we can utilize the algorithmic method to prove lower bounds for them.

We will consider a larger function collection $\mathcal{F}$ containing lowdegree $\mathbb{F}_{2}$-polynomials as a subset, such that the following hold: (1) $\mathcal{F}$ is still simple enough to be analyzed non-trivially by algorithms and (2) it is expressive enough so that one can design a near-optimal $\mathcal{F}$-restrictable generator. Therefore, we can then apply our derandomized XOR Lemma to prove strong average-case lower bounds against $\mathcal{F}$, which immediately implies correlation bounds against $\mathbb{F}_{2}$-polynomials.

The larger function collection $\mathcal{F}$. Formally, fixing an integer $n \in \mathbb{N}$ and let $d=\sqrt{n}$. We define $\mathcal{F}$ as the function collection such that $\mathcal{F}$ consists of all functions $f$ which has at least $n$ input bits, and can be written as $f(x)=(-1)^{P(x)} \cdot g(x)$ for $x \in\{0,1\}^{m}$, where $P$ is an $\mathbb{F}_{2}$-polynomial with degree bounded by $d$ and $g:\{0,1\}^{m} \rightarrow\{-1,1\}$ is a function that only depends on the $(n-d)$-length prefix of its input.

\footnotetext{
${ }^{17}$ To be more precise, we will show that $\mathbb{E}_{w \in\{0,1\}} n k \operatorname{corr}\left(\mathrm{Amp}^{f ; w}-\mathrm{Amp}^{h ; w}, \mathcal{F}_{m}\right)$ is small.
} 
$\mathcal{F}$ is algorithmic friendly. We observe that the fast \#SAT algorithm for low-degree polynomials can be extended to $\mathcal{F}_{m}$-functions naturally. In fact, given a degree- $d$ polynomial $P: \mathbb{F}_{2}^{m} \rightarrow \mathbb{F}_{2}$, we set $\ell=n / d$. For each $\left(x_{1}, \ldots, x_{m-\ell}\right) \in\{0,1\}^{m-\ell}$, we define

$$
s\left(x_{1}, \ldots, x_{m-\ell}\right)=\sum_{\left(y_{1}, \ldots, y_{\ell}\right) \in\{0,1\}^{\ell}}(-1)^{P\left(x_{1}, \ldots, x_{m-\ell}, y_{1}, \ldots, y_{\ell}\right)},
$$

where the above sum is over $\mathbb{Z}$ instead of over $\mathbb{F}_{2}$. Then applying the modulus-amplifying polynomials $([4,50]$, also see the full version for the details), there is an algorithm which can compute the list

$$
\left(s\left(x_{1}, \ldots, x_{m-\ell}\right)\right)_{\left(x_{1}, \ldots, x_{m-\ell}\right) \in\{0,1\}^{m-\ell}}
$$

in $O\left(2^{m-\Omega(\ell)}\right)$ time. Finally, taking a sum over the list, one can then compute $\sum_{x \in\{0,1\}^{m}(-1)^{P(x)}}$ in $2^{m-\Omega(\ell)}$ time.

Now, observing that we have set $\ell=d=\sqrt{n}$ and noting that $g(x)$ only depends on the first $n-d=n-\ell \leq m-\ell$ bits of $x$ (recall that $m \geq n$ ), we have

$$
\begin{aligned}
& \sum_{x \in\{0,1\}^{m}} f(x)= \\
& \sum_{\left(x_{1}, \ldots, x_{m-\ell}\right) \in\{0,1\}^{m-\ell}} s\left(x_{1}, \ldots, x_{m-\ell}\right) \cdot g\left(1, \ldots, x_{n-\ell}, 0, \ldots, 0\right),
\end{aligned}
$$

which allows us to compute $\sum_{x \in\{0,1\}^{m}} f(x)$ in $2^{m-\Omega(\ell)}$ time as well.

The star-like generator for $\mathcal{F}$. Since we aim to prove Theorem 1.1, in the following we fix the number of instances generated by the generator to be $k=\sqrt{n}$. The $\mathcal{F}$-restrictble generator $\mathcal{G}$ is then designed as follows: It has seed length $m=(n-d)+k d \leq 2 n$ (recall that $d=\sqrt{n}$ ). For a seed $r \in\{0,1\}^{m}$, we write $r=\alpha \circ x_{1} \circ \cdots \circ x_{k}$ where $\alpha \in\{0,1\}^{n-d}$ and $x_{1}, \ldots, x_{k} \in\{0,1\}^{d}$. Then $\mathcal{G}$ is defined as follows:

$$
\mathcal{G}(r):=\left(\alpha \circ x_{1}, \ldots, \alpha \circ x_{k}\right) .
$$

Now we can justify why we call it the star-like generator: the $k$ instances generated by $\mathcal{G}$ form a star with their common intersection $\alpha$ as the center. For a string $\alpha \in\{0,1\}^{m-n}$, we write $\alpha=\alpha_{1} \circ \cdots \circ \alpha_{k-1}$ where $\alpha_{i} \in\{0,1\}^{d}$ for every $i \in[k-1]$. For each $i \in[k]$, we define the embedding function $T_{i}$ as

$$
T_{i}(x, \alpha)=\left(x_{\leq n-d}, \alpha_{1}, \ldots, \alpha_{i-1}, x_{>n-d}, \alpha_{i}, \ldots, \alpha_{k-1}\right) .
$$

That is, $T_{i}$ uses $\alpha$ to fill in the length- $d$ suffix for all instances except for the $i$-th one, and use $x$ to fill in the $i$-th instance. It is straightforward to verify that $\mathcal{G}$ satisfies the first two requirements of Definition 2.6.

To show that $\mathcal{G}$ satisfies the third requirement of Definition 2.6, we have to argue that for every $C \in \mathcal{F}_{m}$, (8) is still in $\mathcal{F}_{n}$. Observe that for every $j \in[k] \backslash\{i\}, u_{j}\left(\mathcal{G}\left(T_{i}(x, \alpha)\right)_{j}\right)$ only depends on $x_{\leq n-d}$, hence it belongs to $\mathcal{F}_{n}$. It is also easy to verify that $C\left(T_{i}(x, \alpha)\right) \in \mathcal{F}_{n}$. Since $\mathcal{F}_{n}$ is closed under multiplication, we can conclude that (8) belongs to $\mathcal{F}_{n}$ as well, which completes the proof. (Check the full version for more details)

2.4.2 The Bi-coloring Generator for Constructing Rigid Matrices. Now we turn to the generator for low-rank matrices, which will be used to construct the extremely rigid matrices in Theorem 1.5. First we define the class of "low-rank matrices". For every even $n \geq 1$, we can view a function $f:\{0,1\}^{n}$ as a $2^{n / 2} \times 2^{n / 2}$ matrix, denoted by $M_{f}$, where $M_{f}(x, y)=f(x, y)$ for every $x, y \in\{0,1\}^{n / 2}$. We call the first and last $n / 2$-bits of inputs as the row index and the column index, respectively. Letting $r(n) \geq n^{\omega(1)}$ be the rank parameter, we let $\mathcal{M}_{n}$ denote the class of functions $f$ whose matrix representation $M_{f}$ satisfies $\operatorname{rank}\left(M_{f}\right) \leq r(n)$.

We will construct an $\mathcal{M}$-restrictable generator with seed length $n \sqrt{k}$, which improves upon the trivial seed length of $n k$. More precisely, assuming $\sqrt{k}$ is an integer for simplicity, and letting $t=\sqrt{k}$ and $m=n t$, we choose an arbitrary but fixed injective mapping $\rho:[k] \rightarrow[t] \times[t]$, denoted by $\rho(i)=\left(\rho(i)_{u}, \rho(i)_{v}\right)$. For every $z \in\{0,1\}^{m}$, we write $z=x_{1} \circ \cdots \circ x_{t} \circ y_{1} \circ \cdots \circ y_{t}$ where $\left|x_{i}\right|=\left|y_{j}\right|=n / 2$ for every $i, j \in[t]$. Our generator $\mathcal{G}$ is then defined as

$$
\mathcal{G}(z):=\left(x_{\rho(1)_{u}} \circ y_{\rho(1)_{v}}, \ldots, x_{\rho(k)_{u}} \circ y_{\rho(k)_{v}}\right) .
$$

It is then straightforward to construct the required mappings $T_{i}$ : given $x \in\{0,1\}^{n}$ and $\alpha \in\{0,1\}^{n(t-1)}$, we simply set $x_{\rho(i)_{u}}$ to $x_{\leq n / 2}$ and $y_{\rho(i)_{v}}$ to $x_{>n / 2}$, and use $\alpha$ to fill the rest of $z$. It is easy to verify that $\mathcal{G}$ satisfies the first two requirements of Definition 2.6.

Intuitively, one can interpret the above construction by thinking about a bipartite graph, where the left and right side have $t$ vertices each, and the seed $z$ as a labeling of all vertices by strings in $\{0,1\}^{n / 2}$ (i.e., the strings $x_{1}, \ldots, x_{t}$ and $\left.y_{1}, \ldots, y_{t}\right)$. For every $(i, j) \in[t] \times[t]$, we add an edge between the $i$-th vertex on the left side and the $j$-th vertex on the right side, and label this edge with the concatenation of the two $n / 2$-bit strings on its endpoints (i.e. $x_{i} \circ y_{j}$ ).

Each edge can then be viewed as an instance generated by the seed $z$ (so there are $t^{2} \geq k$ instances in total. Now we argue why $\mathcal{G}$ satisfies the third requirement of Definition 2.6. The crucial property is that every two distinct edges can only share at most one common points. Hence, fixing $i \in[k]$ and $\alpha \in\{0,1\}^{n(t-1)}$, then for $j \neq i$, either $\rho(j)_{u} \neq \rho(i)_{u}$ or $\rho(j)_{v} \neq \rho(i)_{v}$.

We can now observe that, for fixed $\alpha \in\{0,1\}^{n(t-1)}$ and every function $u_{j}:\{0,1\}^{n} \rightarrow\{-1,1\}, u_{j}\left(\mathcal{G}\left(T_{i}(x, \alpha)\right)_{j}\right)$ either only depends on $x_{\leq n / 2}$, or only depends on $x_{>n / 2}$, which means it is a matrix of rank at most 1 . Taking an XOR (multiplication over the $\{-1,1\}$ basis is equivalent to XOR over the Boolean basis) of $k-1$ such matrices resulting in a matrix of rank at most $k-1$. Moreover, one can also observe that for a low-rank matrix $C, C\left(T_{i}(x, \alpha)\right)$ has the same rank as of $C$. Therefore, (8) has low rank too, which completes the argument.

\section{ACKNOWLEDGMENT}

We would like to thank Roei Tell and R. Ryan Williams for many helpful discussions during the project. We are greatful to Emanuele Viola for helpful comments on a draft of this paper. We would like to thank Zhenjian Lu for sharing a manuscript of [31] to us and for comments on an early draft of this paper. We are also grateful to an anonymous reviewer for pointing out an issue in the proof of Theorem 1.5 in an early draft of this paper.

The first author is also grateful to William M. Hoza for pointing out that the Nisan-Zuckerman PRG can be used to improve the seed length in our applications, as well as discussions on pseudodistributions. The second author wants to thank R. Ryan Williams for hosting him during the spring and the summer of 2020 in MIT.

Lijie Chen is supported by an IBM Fellowship. 


\section{REFERENCES}

[1] Josh Alman and Lijie Chen. 2019. Efficient Construction of Rigid Matrices Using an NP Oracle. In Proc. 60th FOCS. IEEE Comp. Soc., 1034-1055. https://doi.org/ 10.1109/FOCS.2019.00067

[2] László Babai, Noam Nisan, and Mario Szegedy. 1992. Multiparty Protocols, Pseudorandom Generators for Logspace, and Time-Space Trade-Offs. 7. Comput System Sci. 45, 2 (1992), 204-232. https://doi.org/10.1016/0022-0000(92)90047-M

[3] Boaz Barak, Moritz Hardt, and Satyen Kale. 2009. The uniform hardcore lemma via approximate Bregman projections. In Proc. 20th Ann. ACM-SIAM Symp. on Discrete Algorithms (SODA'09). ACM Press, 1193-1200. http://dl.acm.org/citation. cfm?id=1496770.1496899

[4] Richard Beigel and Jun Tarui. 1994. On ACC. Comput. Complex. 4 (1994), 350-366. https://doi.org/10.1007/BF01263423

[5] Amey Bhangale, Prahladh Harsha, Orr Paradise, and Avishay Tal. 2020. Rigid Matrices From Rectangular PCPs. In Proc. 61st FOCS. IEEE Comp. Soc. https //eccc.weizmann.ac.il/report/2020/075/

[6] Jean Bourgain. 2005. Estimation of certain exponential sums arising in complexity theory. Comptes Rendus Mathematique 340, 9 (2005), 627-631. https://doi.org/10. 1016/j.crma.2005.03.008

[7] Mark Braverman, Gil Cohen, and Sumegha Garg. 2020. Pseudorandom Pseudodistributions with Near-Optimal Error for Read-Once Branching Programs. SIAM f. Comput. 49, 5 (2020). https://doi.org/10.1137/18M1197734

[8] Eshan Chattopadhyay, Jason Gaitonde, Chin Ho Lee, Shachar Lovett, and Abhishek Shetty. 2020. Fractional Pseudorandom Generators from Any Fourier Level Electron. Colloquium Comput. Complex. 27 (2020), 121. https://eccc.weizmann.ac. $\mathrm{il} /$ report/2020/121

[9] Eshan Chattopadhyay, Pooya Hatami, Kaave Hosseini, Shachar Lovett, and David Zuckerman. 2020. XOR lemmas for resilient functions against polynomials. In Proc. 52nd STOC. ACM Press, 234-246. https://doi.org/10.1145/3357713.3384242

[10] Eshan Chattopadhyay, Pooya Hatami, Shachar Lovett, and Avishay Tal. 2019 Pseudorandom Generators from the Second Fourier Level and Applications to AC0 with Parity Gates. In Proc. 10th Innovations in Theoretical Computer Science Conf. (ITCS'19). Schloss Dagstuhl-Leibniz-Zentrum fuer Informatik, 22:1-22:15. https://doi.org/10.4230/LIPIcs.ITCS.2019.22

[11] Eshan Chattopadhyay and Jyun-Jie Liao. 2020. Optimal Error Pseudodistributions for Read-Once Branching Programs. In Proc. 35th Conf. Computational Complexity (CCC'20). Schloss Dagstuhl-Leibniz-Zentrum fuer Informatik, 25:1-25:27. https //doi.org/10.4230/LIPIcs.CCC.2020.25

[12] Lijie Chen, Xin Lyu, and Ryan Williams. 2020. Almost-Everywhere Circuit Lower Bounds from Non-Trivial Derandomization. In Proc. 61st FOCS. IEEE Comp. Soc. https://eccc.weizmann.ac.il/eccc-reports/2020/TR20-150/

[13] Lijie Chen and Hanlin Ren. 2020. Strong average-case lower bounds from nontrivial derandomization. In Proc. 52nd STOC. ACM Press, 1327-1334. https: //doi.org/10.1145/3357713.3384279

[14] Lijie Chen and R. Ryan Williams. 2019. Stronger Connections Between Circuit Analysis and Circuit Lower Bounds, via PCPs of Proximity. In Proc. 34th Conf. Computational Complexity (CCC'19). Schloss Dagstuhl-Leibniz-Zentrum fuer Informatik, Dagstuhl, Germany, 19:1-19:43. http://drops.dagstuhl.de/opus/ volltexte/2019/10841

[15] Michael A. Forbes and Zander Kelley. 2018. Pseudorandom Generators for ReadOnce Branching Programs, in Any Order. In Proc. 59th FOCS. IEEE Comp. Soc., 946-955. https://doi.org/10.1109/FOCS.2018.00093

[16] Oded Goldreich, Noam Nisan, and Avi Wigderson. 2011. On Yao's XOR-Lemma. In Studies in Complexity and Cryptography. Miscellanea on the Interplay between Randomness and Computation, Oded Goldreich (Ed.). Lecture Notes in Computer Science, Vol. 6650. Springer, 273-301. https://doi.org/10.1007/978-3-642-22670$0 \_23$

[17] Alexander Golovnev, Alexander S. Kulikov, and R. Ryan Williams. 2018. Circuit Depth Reductions. Electron. Colloquium Comput. Complex. 25 (2018), 192. https //eccc.weizmann.ac.il/report/2018/192

[18] Frederic Green, Amitabha Roy, and Howard Straubing. 2005. Bounds on an exponential sum arising in Boolean circuit complexity. Comptes Rendus Mathematique 341, 5 (2005), 279-282. https://doi.org/10.1016/j.crma.2005.07.011

[19] Elad Haramaty, Chin Ho Lee, and Emanuele Viola. 2018. Bounded Independence Plus Noise Fools Products. SIAM F. Comput. 47, 2 (2018), 493-523. https://doi. org/10.1137/17M1129088

[20] Johan Håstad. 1989. Almost Optimal Lower Bounds for Small Depth Circuits. Adv. Comput. Res. 5 (1989), 143-170.

[21] Alexander Healy, Salil P. Vadhan, and Emanuele Viola. 2006. Using Nondeterminism to Amplify Hardness. SIAM 7. Comput. 35, 4 (2006), 903-931. https://doi.org/10.1137/S0097539705447281

[22] Thomas Holenstein. 2005. Key agreement from weak bit agreement. In Proc. 37th STOC. ACM, 664-673. https://doi.org/10.1145/1060590.1060689

[23] Xuangui Huang and Emanuele Viola. 2020. Average-case rigidity lower bounds. Electron. Colloquium Comput. Complex. 26 (2020), 175. https://eccc.weizmann.ac. $\mathrm{il} /$ report/2019/175
[24] Russell Impagliazzo. 1995. Hard-Core Distributions for Somewhat Hard Problems. In Proc. 36th FOCS. IEEE Comp. Soc., 538-545. https://doi.org/10.1109/SFCS.1995. 492584

[25] Russell Impagliazzo and Avi Wigderson. 1997. $P=B P P$ if $E$ Requires Exponential Circuits: Derandomizing the XOR Lemma. In Proc. 29th STOC. ACM Press, 220229. https://doi.org/10.1145/258533.258590

[26] Swastik Kopparty and Srikanth Srinivasan. 2018. Certifying Polynomials for $\mathrm{AC}^{0}[\oplus]$ Circuits, with Applications to Lower Bounds and Circuit Compression. Theory Comput. 14, 1 (2018), 1-24. https://doi.org/10.4086/toc.2018.v014a012

[27] Chin Ho Lee and Emanuele Viola. 2017. More on bounded independence plus noise: Pseudorandom generators for read-once polynomials. Electron. Colloquium Comput. Complex. 24 (2017), 167. https://eccc.weizmann.ac.il/report/2017/167

[28] Leonid A. Levin. 1987. One-way functions and pseudorandom generators. Combinatorica 7, 4 (1987), 357-363. https://doi.org/10.1007/BF02579323

[29] Nutan Limaye, Karteek Sreenivasaiah, Srikanth Srinivasan, Utkarsh Tripathi, and S. Venkitesh. 2019. A fixed-depth size-hierarchy theorem for $\mathrm{AC}^{0}[\oplus]$ via the coin problem. In Proc. 51st STOC. ACM Press, 442-453. https://doi.org/10.1145/ 3313276.3316339

[30] Satyanarayana V. Lokam. 2009. Complexity Lower Bounds using Linear Algebra. Foundations and Trends in Theoretical Computer Science 4, 1-2 (2009), 1-155. https://doi.org/10.1561/0400000011

[31] Zhenjian Lu. 2020. Personal Communication.

[32] Noam Nisan. 1992. Pseudorandom generators for space-bounded computation. Combinatorica 12, 4 (1992), 449-461. https://doi.org/10.1007/BF01305237

[33] Noam Nisan and Avi Wigderson. 1994. Hardness vs Randomness. 7. Comput. System Sci. 49, 2 (1994), 149-167. https://doi.org/10.1016/S0022-0000(05)80043-1

[34] Igor Carboni Oliveira, Rahul Santhanam, and Srikanth Srinivasan. 2019. Parity Helps to Compute Majority. In Proc. 34th Conf. Computational Complexity (CCC'19), Vol. 137. Schloss Dagstuhl-Leibniz-Zentrum fuer Informatik, 23:123:17. https://doi.org/10.4230/LIPIcs.CCC.2019.23

[35] Ninad Rajgopal, Rahul Santhanam, and Srikanth Srinivasan. 2018. Deterministically Counting Satisfying Assignments for Constant-Depth Circuits with Parity Gates, with Implications for Lower Bounds. In Proc. 43rd International Symposium on Mathematical Foundations of Computer Science (MFCS'18), Vol. 117. Schloss Dagstuhl-Leibniz-Zentrum fuer Informatik, 78:1-78:15. https://doi.org/10.4230/ LIPIcs.MFCS.2018.78

[36] Alexander A. Razborov. 1987. Lower bounds on the size of bounded depth circuits over a complete basis with logical addition. Mathematical Notes of the Academy of Sciences of the USSR 41, 4 (1987), 333-338.

[37] Alexander A. Razborov. 1989. On rigid matrices (in Russian). http://people.cs. uchicago.edu/ razborov/files/rigid.pdf Steklov Mathematical Institute.

[38] Omer Reingold, Luca Trevisan, Madhur Tulsiani, and Salil P. Vadhan. 2008. Dense Subsets of Pseudorandom Sets. In Proc. 49th FOCS. IEEE Comp. Soc., 76-85. https://doi.org/10.1109/FOCS.2008.38

[39] Roman Smolensky. 1987. Algebraic Methods in the Theory of Lower Bounds for Boolean Circuit Complexity. In Proc. 19th STOC. ACM Press, 77-82. https: //doi.org/10.1145/28395.28404

[40] Roman Smolensky. 1993. On Representations by Low-Degree Polynomials. In 34th Annual Symposium on Foundations of Computer Science, Palo Alto, California, USA, 3-5 November 1993. IEEE Computer Society, 130-138. https://doi.org/10. 1109/SFCS.1993.366874

[41] Luca Trevisan, Madhur Tulsiani, and Salil P. Vadhan. 2009. Regularity, Boosting, and Efficiently Simulating Every High-Entropy Distribution. In Proc. 24th Conf. Computational Complexity (CCC'09). IEEE Comp. Soc., 126-136. https://doi.org/ 10.1109/CCC.2009.41

[42] Leslie G. Valiant. 1977. Graph-Theoretic Arguments in Low-Level Complexity. In Mathematical Foundations of Computer Science 1977, 6th Symposium, Tatranska Lomnica, Czechoslovakia, September 5-9, 1977, Proceedings (Lecture Notes in Computer Science, Vol. 53). 162-176. https://doi.org/10.1007/3-540-08353-7_135

[43] Emanuele Viola. 2009. Guest Column: correlation bounds for polynomials over $\{0,1\}$. SIGACT News 40, 1 (2009), 27-44. https://doi.org/10.1145/1515698.1515709

[44] Emanuele Viola. 2020. Matching Smolensky's correlation bound with majority. Electron. Colloquium Comput. Complex. 20 (2020), 193. https://eccc.weizmann.ac. $\mathrm{il} / \mathrm{report} / 2020 / 193 /$

[45] Emanuele Viola. 2020. New lower bounds for probabilistic degree and AC0 with parity gates. Electron. Colloquium Comput. Complex. 27 (2020), 15. https: //eccc.weizmann.ac.il/report/2020/015

[46] Emanuele Viola and Avi Wigderson. 2008. Norms, XOR Lemmas, and Lower Bounds for Polynomials and Protocols. Theory of Computing 4, 1 (2008), 137-168. https://doi.org/10.4086/toc.2008.v004a007

[47] Ryan Williams. 2013. Improving Exhaustive Search Implies Superpolynomial Lower Bounds. SIAM 7. Comput. 42, 3 (2013), 1218-1244. https://doi.org/10.1137/ 10080703X

[48] Ryan Williams. 2014. Nonuniform ACC circuit lower bounds. f. ACM 61, 1 (2014), 2. https://doi.org/10.1145/2559903

[49] Andrew Chi-Chih Yao. 1982. Theory and Applications of Trapdoor Functions (Extended Abstract). In Proc. 23rd FOCS. IEEE Comp. Soc., 80-91. https://doi.org/ 
$10.1109 / \mathrm{SFCS} .1982 .45$
[50] Andrew Chi-Chih Yao. 1990. On ACC and Threshold Circuits. In Proc. 31st FOCS. IEEE Comp. Soc., 619-627. https://doi.org/10.1109/FSCS.1990.89583 